\title{
Development of a progressive audiologic tinnitus management program for Veterans with tinnitus
}

\author{
Paula J. Myers, PhD $;^{1 *}$ Susan Griest, MPH $;{ }^{2-3}$ Christine Kaelin, MBA; ${ }^{2}$ Marcia W. Legro, PhD; ${ }^{2}$ Caroline J. \\ Schmidt, PhD; ${ }^{4}$ Tara L. Zaugg, AuD; ${ }^{2}$ James A. Henry, PhD ${ }^{2-3}$

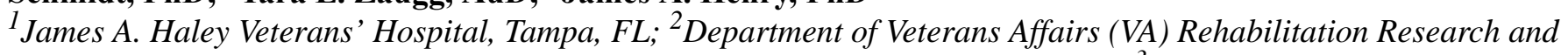 \\ Development, National Center for Rehabilitative Auditory Research, Portland, OR; ${ }^{3}$ Department of Otolaryngology/ \\ Head \& Neck Surgery, Oregon Health \& Science University, Portland, OR; ${ }^{4}$ VA Connecticut Healthcare System, West \\ Haven, CT; and Department of Psychiatry, Yale University School of Medicine, New Haven, CT
}

\begin{abstract}
Tinnitus is the most prevalent service-connected disability awarded to Veterans. However, clinical protocols for management of tinnitus have been inconsistent across Department of Veterans Affairs (VA) medical centers. A study was funded to develop and pilot test a protocol to provide tinnitus services consistently across VA audiology clinics. Drawing on a series of prior VA and external research projects, a clinical model was formulated, supporting materials in multimedia were developed, and a pilot study was conducted. Five hierarchical levels of care were defined and labeled the Progressive Audiologic Tinnitus Management (PATM) model. The model facilitates access to medical services for tinnitus and includes detailed protocols for evaluation, education, and counseling of patients. Patients at each level of care have the option to "progress" to the next level of PATM if further services are required. Clinical procedures were defined for each level and materials were produced for audiologists and patients. The PATM model was then piloted with clinical patients at the James A. Haley Veterans' Hospital (JAHVH) in Tampa, Florida. Throughout the pilot study, feedback from patients and clinicians was carefully noted. Training materials for audiologists, incorporation of the protocol into clinic activities, and patient outcomes were evaluated. The model was implemented within the JAHVH Audiology Clinic and to assist Veterans with tinnitus management. The most notable finding was how little tinnitus-specific intervention was required for the majority of patients. This finding supports a clinical model that offers stepped-care ("progressive") levels of care until tinnitus management is achieved by the patient.
\end{abstract}

Key words: auditory disorders, hearing disorders, outcome and process assessment (health care), pilot projects, Progressive Audiologic Tinnitus Management, tinnitus, tinnitus intervention, treatment outcome, VA audiology, Veterans.

\section{INTRODUCTION}

Tinnitus or "ringing in the ears" affects 10-15 percent of adults in the general population [1], although only a small subset of those with tinnitus are bothered by it to the degree that they seek clinical intervention [2].

\footnotetext{
Abbreviations: $A H R Q=$ Agency for Healthcare Research and Quality, CBT = cognitive-behavioral therapy, HHI = Hearing Handicap Inventory, HHIE-S = Hearing Handicap Inventory for the Elderly-Screening version, IRB = institutional review board, JAHVH = James A. Haley Veterans' Hospital, PATM = Progressive Audiologic Tinnitus Management, RA = research assistant, THI = Tinnitus Handicap Inventory, THS = Tinnitus and Hearing Survey, UC = usual care, VA = Department of Veterans Affairs.

*Address all correspondence to Paula J. Myers, PhD; James A. Haley Veterans' Hospital, Audiology Clinic, 14020 N 46th St, Tampa, FL 33613; 813-972-7529; fax: 813978-5812. Email: paula.myers@va.gov
} http://dx.doi.org/10.1682/JRRD.2013.08.0189 
Patients with a more severe problem with tinnitus are more likely to experience comorbid depression [3], anxiety [4-5], and posttraumatic stress disorder [6] than those with a less severe problem. The prevalence of tinnitus is increasing because of the increasing pervasiveness of toxic noise and other ear-damaging consequences of a highly technological society [7]. There are no guidelines governing the provision of tinnitus clinical servicesleaving the sufferer with tinnitus at a clear disadvantage when seeking care. Although tinnitus is very prevalent among military Veterans (it is the most common serviceconnected disability), many Department of Veterans Affairs (VA) medical centers do not provide systematic clinical services for tinnitus [8].

Since 1995, we have conducted research focusing on different aspects of tinnitus clinical management. In 1999, the team began a series of clinical trials to evaluate different methods of tinnitus management [9]. This work not only provided efficacy data but also identified procedures that were most efficient for clinical application. The first three of these studies [9-11] supported the need for a stepped-care ("progressive") approach to tinnitus management. We designed and conducted a fourth study (reported herein) to develop and pilot test Progressive Audiologic Tinnitus Management (PATM). PATM was developed as a hierarchical model of providing clinical services for Veteran patients who complain of tinnitus [12]. The hierarchy of services involved in the protocol includes five stepped levels of care.

The objective of this study was to define and test a tinnitus-management protocol that could be administered in VA Audiology clinics with minimal impact on routine clinical activities. The work required two phases. For phase 1, a model of care was defined and the necessary materials to administer the protocol were developed. Phase 2 involved conducting a pilot study to examine the feasibility and potential efficacy of the new program.

\section{METHODS}

\section{Phase 1: Development of PATM Model and Supporting Materials}

\section{PATM Model}

A thorough literature review was conducted with particular attention to the prior tinnitus clinical services offered for Veterans. Weekly conferences were held with the study team as the model was developed, drawing on VA and external experts as needed. The proposed tinnitusmanagement program was based on a "progressive intervention" approach that we had previously conceptualized [13]. The program took into account the fact that most individuals with tinnitus do not require extensive intervention [2] (Figure 1). Most of them require education, which can be administered efficiently and consistently through a structured group education process [10,14-15]. A triaging protocol was also required to ensure that the tinnitus does not indicate the presence of a medical problem requiring surgery (a rarity) or some other serious medical condition. The five-level progressive intervention program (Figure 2) used the first three levels to provide services that would meet the needs of the majority of patients complaining of tinnitus: triaging (level 1), auditory assessment and address/rule out medical concerns (level 2), and provision of self-help education in a group setting (level 3). The relatively few patients requiring further services could progress to receive an in-depth tinnitus evaluation (level 4) and individualized intervention as needed (level 5). Appendix 1 (available online only) provides details of the PATM protocol and the clinical materials that were developed.

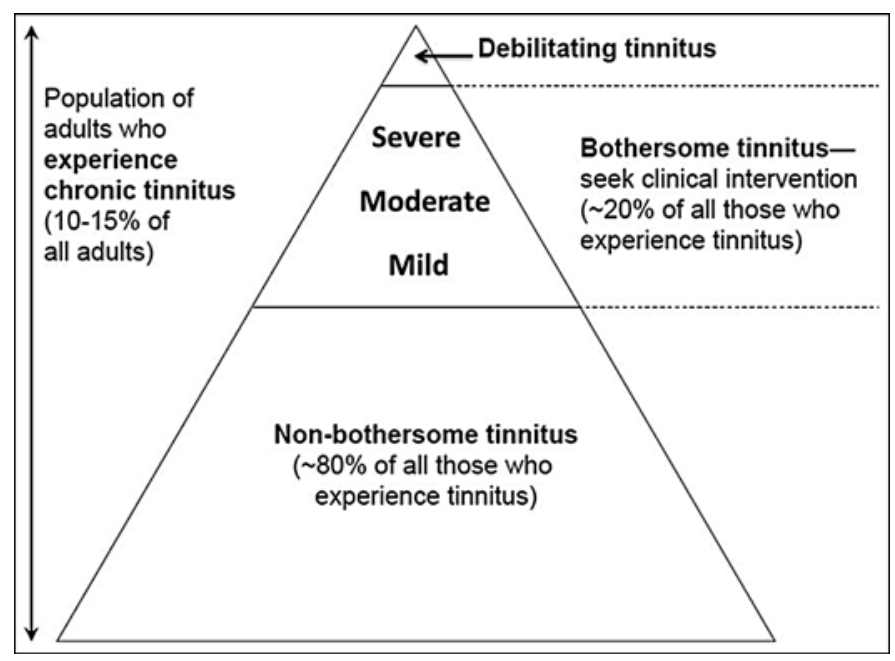

Figure 1.

Tinnitus pyramid [2]. Concept depicted here is that pyramid contains entire population of people who experience chronic tinnitus. Majority of these people (lower part of pyramid) are not particularly bothered by their tinnitus. Many only want assurance that their tinnitus does not reflect some serious medical condition (middle of pyramid). Relatively few have tinnitus that requires some degree of clinical intervention (toward top of pyramid). Very small fraction has "debilitating" tinnitus (top of pyramid). 


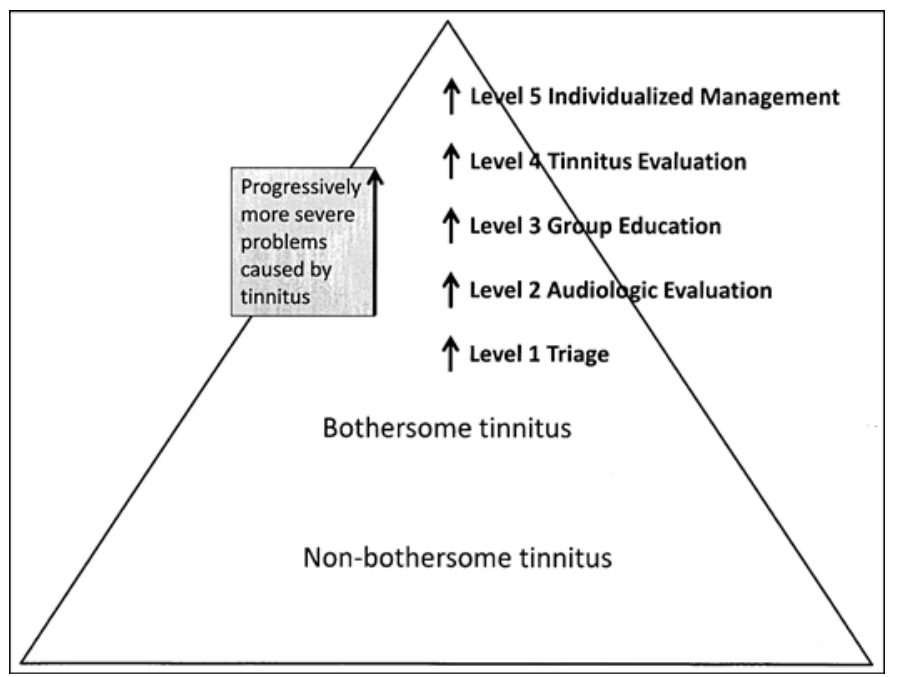

Figure 2.

Tinnitus pyramid represents population of all people who experience chronic tinnitus (see Figure 1). Base of pyramid contains, within this population, largest subgroup whose tinnitus is "nonbothersome." Patients who report "bothersome tinnitus" should be triaged for proper healthcare services (level 1 Triage). Typical progression for patients beyond level 1 Triage is level 2 (Audiologic Evaluation), level 3 (Group Education), level 4 (Tinnitus Evaluation), and level 5 (Individualized Management). Decreasing numbers of patients require the higher levels of services. In fact, the great majority of patients who complain of tinnitus have their needs met at levels 2 and 3 .

\section{Online Training Course for Audiologists}

The American Speech-Language-Hearing Association includes tinnitus management in its scope of practice for audiologists [16], which is justified for many reasons [17-18]. Audiologists were therefore the primary providers of the intervention for this study. Team members (PJM, TLZ, JAH) wrote and reviewed a series of 15 "chapters" that were deemed necessary to educate audiologists about clinical management of tinnitus using the PATM model. The training Web site incorporated a modular design (i.e., individual training modules) and was professionally formatted by Information Technology staff at the James A. Haley Veterans' Hospital (JAHVH) in Tampa, Florida. Plans were made to secure continuing education credits for providers who completed the modules. Details of the online training course are provided in Appendix 2 (available online only).
Multimedia Materials to Support PATM Model in Department of Veterans Affairs Audiology Clinics

Approximately 2 yr were spent developing the PATM clinical materials and online training modules (described in Appendixes 1 and 2, respectively; available online only). This work was guided by the modified PATM model, educational research, and VA guidelines about appropriate language levels and formatting. The materials were repeatedly reviewed and modified by the team of study audiologists.

\section{Phase 2: Pilot Study}

\section{Setting}

The pilot study was embedded into routine clinical care at the JAHVH Audiology Clinic. The study was conducted in compliance with VA policy and with approval from the University of South Florida Institutional Review Board (IRB).

\section{Providers}

Six audiologists at the JAHVH volunteered to conduct the study procedures. All these audiologists had prior experience providing clinical tinnitus services, involving hearing aids, combination instruments (hearing aid and sound generator combined), and basic audiologic counseling (education about tinnitus and various problem-solving and coping strategies). The audiologists were randomized by the study biostatistician to provide either PATM or usual care (UC) for tinnitus management. The three UC audiologists continued to provide their usual tinnitus services, while the three PATM audiologists completed the online training course to conduct PATM. Of note, the audiologists randomized to UC all had considerably more tinnitus-management training and experience than the audiologists randomized to PATM.

\section{Patients}

Study participants were Veteran patients who complained of tinnitus to a provider at the JAHVH. They had to report that their tinnitus was at least a "small problem" (see "Screening and Randomization" section).

\section{Recruitment}

Physicians (Primary Care, Otolaryngology, and Psychiatry) and other healthcare providers at the JAHVH were informed of the study before its implementation. The clinical champion for the study (the JAHVH Chief of 
Audiology Section: PJM) met with the providers and showed them a 14 min patient education video on basic tinnitus management ("Ringing in the Ears: What Can I Do About It?") that was produced by the VA Employee Education System and distributed nationally to all VA hospitals in 2006. The providers received copies of referral guidelines that were developed for the pilot study (Tinnitus Triage Guidelines; see Appendix 1, available online only). None of these providers was required to participate.

Because the study's purpose was to address the "point of care" process (i.e., actual clinical function), recruitment activities were limited to identifying patients with tinnitus from three sources:

1. VA providers who referred patients complaining of tinnitus to the JAHVH Audiology Clinic. The research assistant (RA) called these patients to explain the study and ask whether they were interested in participating.

2. Veterans who called the Audiology Clinic directly to make an appointment. The clinic receptionist inquired whether they had tinnitus (unless they already mentioned it). Patients reporting tinnitus were informed that a research study was open to enrollment and asked whether they would like further information. If so, the RA called them to explain the study and determine whether they were interested in participating.

3 . Veterans who reported tinnitus during a compensation and pension examination for hearing loss and/or tinnitus. They were informed of study enrollment by the examining audiologist and asked whether they would like further information. If so, they received a call from the RA.

These three sources of recruitment were sufficient, and no recruitment advertisements were posted.

\section{Screening and Randomization}

Patients who expressed interest in study participation were asked up to four questions by the RA to determine candidacy: (1) "Do you have ringing, humming, buzzing, or other sounds in your ears or head?" ("yes" response required); (2) "Have you received help for your tinnitus at the Tampa VA?" ("no" required); (3) "If you listen for your tinnitus in a quiet room, can you usually hear it?" ("yes" required); and (4) "Would you say your tinnitus is no problem, a small problem, a moderate problem, a big problem, or a very big problem?" (any choice other than "no problem" required).

Eligible patients who desired to participate in the study were randomized to receive either UC or PATM.
The RA mailed the baseline questionnaires (see "Questionnaires" section) to eligible patients to complete before their initial visit. These patients were not required to sign an informed consent form. A Waiver of Documentation of Informed Consent was approved by the IRB because the clinical services provided in conjunction with the study were considered routine and customary care. (The procedures developed for PATM were evidence based and did not have any more risk than UC.)

\section{Initial Visit}

Upon arrival at the Audiology Clinic, patients first met with the RA or the Chief of Audiology Section (PJM), who collected the baseline questionnaires. Patients then met with an audiologist from the respective group (UC or PATM) to which they were randomized. Patients in both groups underwent audiologic testing according to JAHVH's usual clinical protocol. They were issued amplification and/or other assistive listening devices if indicated to manage hearing problems.

\section{Usual Care Protocol}

After audiologic testing, patients randomized to UC received a frequently asked questions handout on tinnitus education and brief counseling about the use of sound to manage tinnitus. Individualized tinnitus counseling was offered as an option to those patients requesting further services. These patients also had the option of receiving sound therapy devices, including ear-level sound generators and combination instruments, and tabletop sound conditioners.

\section{PATM Protocol}

During the audiologic assessment, all patients randomized to PATM received a self-help workbook that was developed to support PATM [19]. They were told that if they desired further services after reading the workbook, they were welcome to attend two group workshops, separated by $2 \mathrm{wk}$ and conducted by one of the three PATM audiologists. (The workshops and workbook are described in Appendix 1, available online only.) Following the workshops, patients were offered further intervention, if needed.

\section{Questionnaires}

Questionnaires included the Hearing Handicap Inventory (HHI) for the Elderly-Screening Version (HHIE-S) [20], Tinnitus Handicap Inventory (THI) [21-22], and 
Tinnitus and Hearing Survey (THS) [23]. These questionnaires were mailed to all eligible patients following the telephone screening and again 6 mo later. The questionnaires were used clinically to assess each patient's auditory needs before and after receiving audiology services.

The HHI is a statistically validated questionnaire used to measure patients' self-perceived hearing handicap. Different versions of the HHI are available to assess patients $\geq 65 \mathrm{yr}$ [24] and patients $<65 \mathrm{yr}$ [25]. Screening versions of the HHI are also available. For this study, the 10-item HHIE-S was utilized for all subjects [20]. The HHIE-S was chosen because it is short and contains no questions about employment, making it applicable to all patients regardless of employment status and age.

The THI is a statistically validated tinnitus questionnaire that provides an index score, ranging from 0 to 100 , with higher scores reflecting greater self-perceived tinnitus handicap. A change in the index score of at least 20 points is reported to indicate a clinically significant improvement for individuals at the 95 percent confidence level [26]. A shortcoming of the THI (and of all statistically validated tinnitus questionnaires) is that it is vulnerable to influence from hearing problems; i.e., it is not possible to know how much of the score on the THI is due to hearing problems erroneously blamed on tinnitus [27]. Therefore, a high score on the THI could indicate the need for intervention for a hearing problem rather than for a tinnitus problem. For this reason, the THI could not be relied upon alone to determine the need for intervention specifically for tinnitus and was thus supplemented by the THS.

The THS was developed to distinguish between selfperceived tinnitus problems and hearing problems, making it an efficient tool to determine whether intervention specific to tinnitus is needed [23]. Discussion of the THS results with the study subjects was the primary means of determining whether intervention for tinnitus should be offered.

\section{Quantitative Data Analysis}

The THI was the primary outcome measure, and patients in the PATM group were expected to have equal or better tinnitus management as reflected in the mean THI scores. Data were also analyzed to determine whether patients in each group were similar with respect to demographics, health status, and tinnitus severity at baseline. To compare groups, a Student $t$-test was used for continuous variables and a chi-square test for categorical data. Due to multiple comparisons, Bonferroni corrections were applied.

\section{Formative Data Analysis}

Due to the exploratory nature of this project, formative data collection was planned. A formative evaluation has been defined as "a rigorous assessment process designed to identify potential and actual influences on the progress and effectiveness of implementation efforts" [28, p. 2]. Formative data included semistructured surveys for the audiologists about the online training experience, four interviews with the study audiologists as a group during the study, individual discussions between audiologists and the Chief of Audiology Section (PJM) on site throughout the study, and weekly conference calls with the study team for which detailed minutes were taken. Records were kept and data were collated and analyzed by the team implementation specialist (MWL) and reported to the team to identify barriers to care and issues encountered when using the new materials. Themes were noted and changes effected due to this information were recorded.

\section{RESULTS}

\section{Phase 1: Development of PATM Model and Materials}

A clinical handbook was completed that consisted of detailed procedures, checklists, handouts, forms, and questionnaires needed to conduct each of the five hierarchical levels of PATM. A patient self-help workbook with a DVD and $\mathrm{CD}$ was also developed, as was an online training program for the PATM audiologists. The five levels of care and the materials developed for each level are described in Appendix 1 (available online only), and a description of the online training program is provided in Appendix 2 (available online only).

After completing the online training course, audiologists responded anonymously to brief semistructured surveys about the training modules. Questions addressed time to complete, utility of information conveyed, confidence of learner to perform the described practices, and perceived barriers to completing the described practices. Responses were tallied and shared with the study team.

The results of this survey indicated that time to complete each varied by learner and by content from less than $30 \mathrm{~min}$ to more than $2 \mathrm{~h}$ (usually $31-60 \mathrm{~min}$ ). All three PATM audiologists endorsed the course as useful to their practice and that the module content met their stated objectives. Their level of confidence in the ability to perform practices as described was divided between 5175 percent and $76-100$ percent. No one reported seeing 
significant barriers to performing the work. Each PATM audiologist $(n=3)$ received $30-35 \mathrm{~h}$ in $3 \mathrm{~h}$ increments of release time from clinical duties to complete the online course. Each module, or chapter, stated objectives and then presented learning content that also contained comprehension questions throughout. Six final questions were asked to evaluate the module from the learner's perspective. Responses were analyzed and a detailed report was written by the implementation research specialist (MWL). The responses are summarized below [along with lessons we have drawn from them].

- We received responses from about 80 percent of the modules that we know were completed (43 evaluations of 45 completed modules -15 modules $\times 3$ learners) from the online course manager; the course was in late stages of development. [In the next study, the course will be managed by VA Employee Education System personnel, so this gap in records is not expected.]

- Learners estimated they spent a median of "61-90 minutes" to complete a module. However, time required for a module varied by learner and module, with five reports of taking "over 2 hours." [It is necessary for clinic administrators to set aside adequate time for clinicians to complete necessary portions of the course. The course also is being revised with length as well as content in mind and is being approved for audiologists and psychologists to receive continuing education credits.]

- Learners all said they "agree" or "strongly agree" that the information learned would be useful in their practice. [The content of the chapters was relevant to the audiologists.] They also "agree(d)" or "strongly agree(d)" that the content of each module met the stated objectives for that module. [The module content seemed to meet authors' objectives.]

- The confidence level expressed by learners about being able to apply the material in each chapter was evenly split between " $51 \%-75 \%$ " and " $76 \%-100 \%$." [Because half the time learners reported less than high confidence, the implementation consultant (MWL) conducted a series of conference calls with the study team and PATM audiologists throughout the pilot study. The purpose of the calls was to support audiologists' work by answering questions they had and helping to address barriers they encountered. These calls are reported in the next section.]
- The last question at the end of each module asked learners to identify any barriers they foresaw to completing the practices described in the module. Most responses were "none" or the item was left blank. Only one audiologist suggested for one module that there could be a time constraint problem. [The learners judged PATM feasible for their practice.]

\section{Phase 2: Pilot Study}

\section{Study Patients}

Following self-referral or referral by a provider, 221 patients at the JAHVH Audiology Clinic with a complaint of tinnitus were contacted by telephone and recruited; 109 were randomized to UC and 112 to PATM. Of these 221 patients, 20 randomized to $\mathrm{UC}$ and 20 randomized to PATM were excluded from participation because they did not show up, called to be removed, or had a serious health concern that might have interfered with participation. A total of 92 PATM patients and 89 UC patients completed questionnaires and attended the initial visit.

The randomization was successful in that patients in the PATM and UC groups were not significantly different in demographics (age, sex, race, education) or health variables (perceived health, mental health diagnoses, or tinnitus handicap) (Table). The patients randomized to PATM varied widely by age, averaging $56 \mathrm{yr}$. They were predominantly male and half reported education beyond grade 12 .

Table.

Study patient characteristics.

\begin{tabular}{|c|c|c|c|}
\hline Characteristic & $\begin{array}{c}\text { PATM } \\
(n=92)\end{array}$ & $\begin{array}{c}\text { Usual Care } \\
(n=89)\end{array}$ & Significance \\
\hline \multicolumn{4}{|l|}{ Age (yr) } \\
\hline Mean \pm SD & $56.0 \pm 11.2$ & $59.0 \pm 10.5$ & $\mathrm{NS}^{*}$ \\
\hline Range & $23-87$ & $27-78$ & \\
\hline Sex (\% Male) & 94 & 97 & $\mathrm{NS}^{\dagger}$ \\
\hline Race (\% White) & 78.8 & 81.6 & $\mathrm{NS}^{*}$ \\
\hline Education ( $\%>$ Grade 12$)$ & 57.6 & 65.9 & $\mathrm{NS}^{*}$ \\
\hline \multicolumn{4}{|l|}{ Perceived Health } \\
\hline Very Good or Excellent & 24.5 & 29.9 & $\mathrm{NS}^{*}$ \\
\hline Mental Health Diagnosis & 41.3 & 38.2 & $\mathrm{NS}^{\dagger}$ \\
\hline \multicolumn{4}{|l|}{ THI at Baseline } \\
\hline Mean \pm SD & $37.0 \pm 24.3$ & $43.0 \pm 25.4$ & $\mathrm{NS}^{\dagger}$ \\
\hline Range & $0-100$ & 4-100 & \\
\hline \multicolumn{4}{|c|}{$\begin{array}{l}\dagger \text { Student } t \text {-test (Bonferroni correction: } p=0.05 / 7=0.007 \text { ). } \\
\mathrm{NS}=\text { not significant, PATM = progressive audiologic tinnitus management } \\
(\text { program), } \mathrm{SD}=\text { standard deviation, THI }=\text { Tinnitus Handicap Inventory. }\end{array}$} \\
\hline
\end{tabular}


Approximately one-quarter of the PATM patients were nonwhite. Their self-reported health ranged from poor to excellent, with 24.5 percent reporting good to excellent heath. Their baseline mean THI index score was 37.0.

\section{Levels of Care Received by Patients}

Of the 92 PATM patients, 28 (30.4\%) chose to receive further care by attending the first level 3 Group Education meeting and $8(8.7 \%)$ attended both sessions. Following level 3, 4 (4.3\%) PATM patients attended a level 4 Tinnitus Evaluation, and only $1(1.1 \%)$ of these received level 5 Individualized Management. Of the 89 UC patients, 37 attended a tinnitus-evaluation appointment and 52 attended a second appointment to receive hearing aids.

\section{Patient Outcome Data}

The THI was completed by 58 of the 92 PATM patients at both baseline and 6 mo. The mean baseline THI index score for these 58 patients was 35.1 and the mean 6 mo score was 33.3 (mean reduction of 1.8 points). The THI was completed by 68 of the 89 UC patients at both baseline and 6 mo. The mean baseline and 6 mo THI index scores for these 68 patients was 42.8 and 39.8, respectively (mean reduction of 3.0 points). We had planned to compare mean THI scores between PATM patients who completed level 3 Group Education with patients who received UC. However, because only 8 of the 92 PATM patients attended both sessions of the level 3 intervention, a statistical comparison of patients between groups would not have yielded meaningful data.

\section{Formative Evaluations}

The three PATM audiologists were interviewed as a group four times during the study about using the PATM model and about the materials provided for this purpose including implementation, acceptance by patients and staff, and clinical effectiveness. They were also asked about satisfaction and the perceived clinical effectiveness of the interventions. Participants on these calls included the implementation consultant, study team members, and the PATM audiologists. A semistructured discussion was conducted on each call, with detailed minutes transcribed for analysis. The following issues were identified and addressed.

- General satisfaction and acceptance by audiologists of the protocol. All three clinicians reiterated their positive experiences delivering care under the PATM protocol. They endorsed the online course and the study materials that were provided.

- Barriers to performing each level of care in the PATM protocol were explored.

- Level 1 Triage. There was a regular stream of referrals for tinnitus management to the JAHVH Audiology Clinic. No one on the program evaluation calls identified barriers to referrals or to scheduling appointments for tinnitus care.

- Level 2 Audiologic Evaluation. Audiologists did not mention any difficulty with completing these evaluations nor barriers to the referrals of their patients for hearing aids (as needed by some patients). Hearing aids were available and provided to the patients. Likewise, the audiologists did not encounter problems reconnecting with patients following acquisition of hearing aids. All patients at this level were provided a self-management workbook; no problems were reported with securing, storing, or handing out the workbooks.

- Level 3 Group Education. Several barriers became apparent at the next step when patients expressed a need for further intervention for their tinnitus, thus beginning group education. Both a serious parking problem at the hospital that affected patient access and willingness to attend sessions and the limited times available for scheduling the two classes $(2 \mathrm{wk}$ apart) presented a problem. Fortunately, the parking situation was since alleviated because the clinic itself was moved to another location with adequate parking.

- Level 4 Tinnitus Evaluation. This level was seldom needed, but when it was, the audiologists did not encounter barriers to this care. No barriers to (or seamlessness of) referrals to mental health or to otolaryngology were mentioned on the calls.

- Level 5 Individualized Management. This level of care was even less likely to be recommended, and it was not a problem to perform the one-on-one counseling.

Weekly telephone conferences were planned between study team members, including the principal investigators (JAH and PJM), study administrator (CK), implementation consultant (MWL), and others as necessary, including the PATM audiologists. Notes were kept about issues discussed and decisions reached. Of primary interest was identifying changes that may be necessary or barriers to performing the work as described. PATM audiologists provided formative data to the co-principal investigator (PJM) 
on an ongoing basis, which focused on improving fidelity to the protocol.

Finally, a post hoc interview was conducted with the Service Chief for Audiology and Speech Pathology at the JAHVH to learn how the study affected the clinic. Themes that appeared from the data included the importance of working with administrators at sites planning to use PATM concerning the requirements of audiologists and patients: release time for audiologists for educational course, to establish a smooth referral system in and out of the audiology clinic, to clarify the kinds and amounts of ear-level devices that may be needed, and to establish the importance of providing a location and time for level 3 classes.

\section{DISCUSSION}

This project was designed to develop all materials necessary to implement the PATM protocol and then to introduce the PATM clinical model at one VA site, obtaining pilot data to evaluate its clinical utility relative to UC (i.e., VA audiology services typically provided to Veterans for tinnitus). A major goal of this project was to learn about the process of putting the PATM protocol into clinical practice. To do so, we conducted several formative evaluations.

\section{Creation of PATM}

Development of the PATM implementation materials was a substantial undertaking requiring 2 yr of continuous effort by the study team. Insights gained from our previous controlled studies were applied to the new PATM protocol. Five hierarchical levels of PATM were defined, and detailed clinical procedures were developed for each level. A completely new counseling protocol was developed that incorporated principles of patient education and health literacy to ensure that the materials were accessible to as many Veterans as possible [29]. An online, 15-module training course was developed for audiologists (see Appendix 2, available online only), and numerous clinical materials were developed for audiologists and patients (see Appendix 1, available online only). Major educational materials were developed to implement the protocol at the different levels, including (1) a self-help workbook (94 pages) given to patients at level 2 Audiologic Evaluation [19], (2) PowerPoint presentations for use during the two classes comprising level 3 Group Education, (3) DVD video presentations for use during the two classes comprising level 3 Group Education, and (4) a counseling guide to facilitate one-on-one counseling during level 5.

\section{PATM Pilot Study}

Following development of the PATM program, a pilot clinical trial of the new method was implemented. The pilot trial did not result in useful outcome data because too few of the patients progressed to receive the intervention (they mostly received an audiologic assessment, and many required amplification). The most notable finding gleaned from this pilot study was how little tinnitus-specific intervention was required for the majority of patients. This finding supports the rationale for progressively providing only as much intervention as needed.

Some comments are necessary to provide the proper perspective to interpret the findings of the pilot study. Normally, a controlled clinical study would involve stringent inclusion/exclusion criteria to ensure that only appropriate subjects are identified and enrolled. If an intervention is offered, then it would be essential to include only subjects who have a condition warranting receipt of the intervention. The "condition" of tinnitus does not necessarily require intervention. It is generally accepted that only about 20 percent of individuals who experience chronic tinnitus consider it to be a significant problem requiring intervention [30-32]. For the present study, inclusion criteria were very lenient to ensure that all patients reporting tinnitus at the JAHVH had the opportunity to receive services that would be consistent with a clinical model. This was a point-of-care study; i.e., the study was embedded into standard clinical care with the exception that patients were randomized to receive one of two approaches of tinnitus management: PATM or UC. The patients who were enrolled only had to claim the presence of tinnitus and indicate that it was at least a "small" problem. These patients represent a typical VA clinic population of Veterans who experience chronic tinnitus and generally would not claim that it significantly impacts their life. The very low number of patients who desired further services supports the basic premise that most patients with tinnitus do not require tinnitusspecific services.

For this study, a waiver of informed consent was obtained so that patients who were enrolled as study subjects did not undergo the formal consenting process that is normally required for controlled clinical studies. Subjects were not held responsible to fulfill certain study 
tasks and compensation was not offered - they were treated as clinic patients and were free to choose to receive or not to receive additional tinnitus services that were offered. The great majority of patients did not choose to receive additional services. Even those PATM patients who went on to attend the level 3 group sessions did not generally return for the second session that was offered. A further challenge for these patients was the unavailability of parking at the JAHVH. The study was conducted at a time when the JAHVH Audiology Clinic was being relocated to a new building miles away from the JAHVH. During the study, the relocation had not yet taken place, and parking at the JAHVH posed a frustrating challenge for Veteran patients. It was clear that many of the patients in our study chose not to return to the JAHVH simply because of the parking problem. This problem has since been resolved because parking is plentiful at the new building.

It was described earlier that six audiologists were randomized to provide either UC or PATM, which coincidentally resulted in placement of audiologists with the most tinnitus-management experience in the UC group. The three UC audiologists also had recently completed online tinnitus-management coursework toward fulfillment of their clinical doctorate degree. Further, two of the three PATM audiologists were not comfortable leading a class (due to shy personalities) and the third was observed to be impatient with tinnitus counseling. The three PATM audiologists therefore did not represent true clinical practice whereby an audiologist comfortable with leading workshops and motivated to patiently administer specialized counseling would be selected to provide tinnitus management. Following randomization of providers, it was recognized that these concerns could potentially confound the results.

A final issue worth noting is the nature of the training provided to the PATM audiologists. As previously described, they completed extensive online training. A team member (PJM) answered questions and was available for debriefing sessions; however, these audiologists did not receive direct supervision to assure fidelity of their intervention. Following the pilot study, we initiated a new protocol to augment the online training: video recordings were made of new clinicians providing the intervention, which were then watched by a PATM expert who provided feedback and guidance as needed. This "remote supervision" process has proven effective in ensuring that the intervention is delivered properly.
Although this pilot study did not result in quantifiable outcome data, the process of developing PATM and pilot testing it in a point-of-care setting has been invaluable for the development of a tinnitus-management program that would be appropriate for VA-wide implementation. The protocol has since been revised and is currently being evaluated in a multi-VA-site randomized clinical study.

\section{Toward More "Evidence-Based" Tinnitus Management}

Most interventions for tinnitus focus on reducing reactions to tinnitus rather than attempting to mitigate the perception [2]. Empirically supported behavioral interventions typically involve variations of therapeutic sound, counseling, coping techniques, stress reduction, and tinnitusspecific education [29]. No one method has been proven superior, although it has been observed that patients typically gain some degree of benefit by receiving a tinnitus assessment, some basic counseling, and the appropriate use of hearing aids [33]. It is likely that no one form of intervention is effective for all those with tinnitus; i.e., there is considerable variability in how patients respond to different interventions [34]. Only a handful of methods have an evidence basis, i.e., have sufficient support in the literature to be considered viable clinical techniques. These methods include tinnitus masking [35-36], tinnitus retraining therapy [37-38], neuromonics tinnitus treatment [39-40], and cognitive-behavioral therapy (CBT) [41-42]. Ear-level devices that are commonly used for tinnitus management include hearing aids [43-44], sound generators [45-46], and combination instruments [46-47].

One of the best guides for the evidence basis of different therapies is the Cochrane Reviews. These systematic reviews of primary research in healthcare are generally thought to reflect the highest standard in evidence-based care for medical conditions. A number of Cochrane Reviews have been published addressing different methods of tinnitus intervention. From one of the most recent reviews, it has been concluded that CBT can improve quality of life and depression scores [48]. Sound therapy studies are more limited. A Cochrane Review concluded that sound therapy studies have generally been of low quality and have not shown that sound therapy on its own provides significant benefit [49]. The authors concluded, however, that "the absence of conclusive evidence should not be interpreted as evidence of lack of effectiveness," and "optimal management may involve multiple strategies" [49, p. 2]. 
The Agency for Healthcare Research and Quality (AHRQ) conducts comparative effectiveness reviews to help healthcare policy makers make evidence-based decisions to improve the quality of healthcare services. The AHRQ recently completed a review for tinnitus with the overall conclusion that none of the methods reviewed had significant strength of evidence [50]. With respect to psychological/behavioral interventions, there was insufficient information to determine the strength of evidence for sound therapies and there was low-quality evidence based on 10 studies (498 participants) that CBT has a beneficial effect on quality of life. The review pointed out that CBT is an indistinct therapeutic method with no strict definition and that CBT was commonly combined with other behavioral interventions. One of their conclusions was "The development of progressive or staged treatments is an active area of interest in the tinnitus field, and this may be a promising avenue for further exploration in future studies."

\section{CONCLUSIONS}

Results of this pilot study suggest that many Veterans who complain of tinnitus do not desire clinical services beyond a basic audiologic evaluation and the fitting of hearing aids if indicated. This same finding has been reported by numerous VA audiologists who have implemented PATM in their clinic. Combined, these research and clinical findings support the principle that tinnitus services should be progressive and should normally start with an audiologic evaluation.

Proper training of clinicians to learn how to provide tinnitus clinical services is essential. Such training is not normally offered in graduate programs, either for audiologists or mental health providers. Numerous books about tinnitus are available; however, most of these books describe a wide variety of approaches without focusing on evidence-based methods. And, in fact, the evidence basis of different methods is equivocal according to the best reports currently available (AHRQ review and Cochrane reviews) [48-50]. The limited evidence available would suggest that tinnitus management should include collaborations with mental health professionals who can offer additional tinnitus coping strategies using CBT. In addition to CBT, patients should be educated about the various uses of sound as therapy for tinnitus. While many sound-based methods have been espoused, and are being marketed, comparative effectiveness studies are needed. Until such studies are conducted, the use of sound therapy should be eclectic and patients should learn how to use sound adaptively to address different tinnitus-problem situations. Patients also need this information to make informed decisions about specialized sound therapy devices and methods that are increasingly becoming available.

\section{ACKNOWLEDGMENTS}

\section{Author Contributions:}

Study concept and design: P. J. Myers, J. A. Henry, T. L. Zaugg, C. Kaelin.

Acquisition of data: P. J. Myers, M. W. Legro.

Drafting of manuscript: P. J. Myers, J. A. Henry, M. W. Legro.

Critical revision of manuscript for important intellectual content: P. J. Myers, S. Griest, C. Kaelin, M. W. Legro, C. J. Schmidt, T. L. Zaugg, J. A. Henry.

Statistical analysis: S. Griest.

Obtained funding: J. A. Henry, P. J. Myers.

Study supervision: J. A. Henry, P. J. Myers.

Administrative support: C. Kaelin.

Financial Disclosures: The authors have declared that no competing interests exist.

Funding/Support: This material was based on work supported by the VA, Rehabilitation Research and Development Service (grants C4488R, C3469S, F7070S). Administrative support and facilities were provided by the JAHVH.

Additional Contributions: We are grateful to Stephen Fausti, $\mathrm{PhD}$, and Dave Hickam, MD, for their valuable consultation services to develop protocols used in this project. Special thanks to Pat Feeney, $\mathrm{PhD}$; John McDermott, PhD; and Sara Ruth O'Dell, AuD, for their significant contributions to our tinnitus research.

Institutional Review: Patients were not required to sign an informed consent form. A Waiver of Documentation of Informed Consent was approved by the IRB because the clinical services provided in conjunction with the study were considered routine and customary care. Participant Follow-Up: The authors do not plan to inform participants of the publication of this study.

Disclaimer: The contents of this publication do not represent the views of the VA or the U.S. Government.

\section{REFERENCES}

1. Hoffman HJ, Reed GW. Epidemiology of tinnitus. In: Snow JB, editor. Tinnitus: Theory and management. Lewiston (NY): BC Decker Inc; 2004. p. 16-41.

2. Dobie RA. Overview: Suffering from tinnitus. In: Snow JB, editor. Tinnitus: Theory and management. Lewiston (NY): BC Decker Inc; 2004. p. 1-7. 
3. Folmer RL, Shi YB. SSRI use by tinnitus patients: Interactions between depression and tinnitus severity. Ear Nose Throat J. 2004;83(2):107-8,110,112 passim. [PMID:15008444]

4. Heath I. Tinnitus and health anxiety. Br J Nurs. 1994;3(10): 502-5. [PMID:8038554]

5. Hesser H, Andersson G. The role of anxiety sensitivity and behavioral avoidance in tinnitus disability. Int $\mathrm{J}$ Audiol. 2009;48(5):295-99. [PMID:19842804]

http://dx.doi.org/10.1080/14992020802635325

6. Fagelson MA. The association between tinnitus and posttraumatic stress disorder. Am J Audiol. 2007;16(2):107-17. [PMID:18056879] http://dx.doi.org/10.1044/1059-0889(2007/015)

7. Gilles A, De Ridder D, Van Hal G, Wouters K, Kleine Punte A, Van de Heyning P. Prevalence of leisure noiseinduced tinnitus and the attitude toward noise in university students. Otol Neurotol. 2012;33:899-906.

8. Henry JA, Zaugg TL, Myers PJ, Schmidt CJ, Griest S, Legro MW, Kaelin C, Thielman EJ, Storzbach DM, McMillan GP, Carlson KF. Pilot study to develop telehealth tinnitus management for persons with and without traumatic brain injury. J Rehabil Res Dev. 2012;49(7):1025-42.

[PMID:23341277] http://dx.doi.org/10.1682/JRRD.2010.07.0125

9. Henry JA, Zaugg TL, Myers PM, Kendall CJ. Progressive tinnitus management: Clinical handbook for audiologists. San Diego (CA): Plural Publishing; 2010.

10. Henry JA, Loovis C, Montero M, Kaelin C, Anselmi KA, Coombs R, Hensley J, James KE. Randomized clinical trial: Group counseling based on tinnitus retraining therapy. J Rehabil Res Dev. 2007;44(1):21-32. [PMID:17551855] http://dx.doi.org/10.1682/JRRD.2006.02.0018

11. Henry JA, Schechter MA, Zaugg TL, Griest S, Jastreboff PJ, Vernon JA, Kaelin C, Meikle MB, Lyons KS, Stewart BJ. Outcomes of clinical trial: Tinnitus masking versus tinnitus retraining therapy. J Am Acad Audiol. 2006;17(2): 104-32. [PMID:16640064] http://dx.doi.org/10.3766/jaaa.17.2.4

12. Henry JA, Zaugg TL, Myers PJ, Schechter MA. Progressive audiologic tinnitus management. ASHA Lead. 2008; 13:14-17.

13. Henry JA, Schechter MA, Loovis CL, Zaugg TL, Kaelin C, Montero M. Clinical management of tinnitus using a "progressive intervention" approach. J Rehabil Res Dev. 2005; 42(4 Suppl 2):95-116. [PMID:16470467] http://dx.doi.org/10.1682/JRRD.2005.01.0005

14. Newman CW, Sandridge SA. Incorporating group and individual sessions into a tinnitus management clinic. In: Tyler RS, editor. Tinnitus treatment: Clinical protocols. New York (NY): Thieme; 2005. p. 187-97.
15. Sandridge SA, Newman CW. Benefits of group informational counseling. In: Dauman R, editor. VIIIth International Tinnitus Seminar; 2005 Sep; Pau, France. Bordeaux (France): ENT Department, University Hospital of Bordeaux; 2005. p. 106.

16. American Speech-Language-Hearing Association. Scope of practice in audiology [Internet]. 2004. Rockville (MD): ASHA. Available from: www.asha.org/policy

17. Henry JA. Audiologic assessment of the tinnitus patient. In: Snow JB, editor. Tinnitus: Theory and management. Lewiston (NY): BC Decker, Inc; 2004. p. 220-36.

18. Henry JA, Zaugg TL, Schechter MA. Clinical guide for audiologic tinnitus management I: Assessment. Am J Audiol. 2005;14(1):21-48. [PMID:16180968] http://dx.doi.org/10.1044/1059-0889(2005/004)

19. Henry JA, Zaugg TL, Schechter MA, Myers PJ. How to manage your tinnitus: A step-by-step workbook. Portland (OR): VA National Center for Rehabilitative Auditory Research; 2008.

20. Lichtenstein MJ, Bess FH, Logan SA. Diagnostic performance of the hearing handicap inventory for the elderly (screening version) against differing definitions of hearing loss. Ear Hear. 1988;9(4):208-11. [PMID:3169401] http://dx.doi.org/10.1097/00003446-198808000-00006

21. Newman CW, Jacobson GP, Spitzer JB. Development of the Tinnitus Handicap Inventory. Arch Otolaryngol Head Neck Surg. 1996;122(2):143-48. [PMID:8630207] http://dx.doi.org/10.1001/archotol.1996.01890140029007

22. Newman CW, Sandridge SA, Jacobson GP. Psychometric adequacy of the Tinnitus Handicap Inventory (THI) for evaluating treatment outcome. J Am Acad Audiol. 1998; 9(2):153-60. [PMID:9564679]

23. Henry JA, Zaugg TL, Myers PJ, Schechter MA. The role of audiologic evaluation in progressive audiologic tinnitus management. Trends Amplif. 2008;12(3):170-87. [PMID:18628281] http://dx.doi.org/10.1177/1084713808319941

24 . Ventry IM, Weinstein BE. Identification of elderly people with hearing problems. ASHA. 1983;25(7):37-42. [PMID:6626295]

25. Newman CW, Weinstein BE, Jacobson GP, Hug GA. The Hearing Handicap Inventory for Adults: Psychometric adequacy and audiometric correlates. Ear Hear. 1990;11(6): 430-33. [PMID:2073976] http://dx.doi.org/10.1097/00003446-199012000-00004

26. Newman CW, Sandridge SA. Tinnitus questionnaires. In: Snow JB, editor. Tinnitus: Theory and management. Lewiston (NY): BC Decker Inc; 2004. p. 237-54.

27. Zaugg TL, Schechter MA, Fausti SA, Henry JA. Difficulties caused by patients' misconceptions that hearing problems are due to tinnitus. In: Patuzzi R, editor. Proceedings of the Seventh International Tinnitus Seminar; 2002; Crawley 
(Australia): The University of Western Australia; 2002. p. 226-28.

28. Stetler CB, Legro MW, Wallace CM, Bowman C, Guihan M, Hagedorn H, Kimmel B, Sharp ND, Smith JL. The role of formative evaluation in implementation research and the QUERI experience. J Gen Intern Med. 2006;21(Suppl 2): S1-8. [PMID:16637954] http://dx.doi.org/10.1007/s11606-006-0267-9

29. Henry JA, Zaugg TL, Myers PJ, Kendall CJ, Turbin MB. Principles and application of educational counseling used in progressive audiologic tinnitus management. Noise Health. 2009;11(42):33-48. [PMID:19265252] http://dx.doi.org/10.4103/1463-1741.45311

30. Davis A, Refaie AE. Epidemiology of tinnitus. In: Tyler R, editor. Tinnitus handbook. San Diego (CA): Singular Publishing Group; 2000. p. 1-23.

31. Jastreboff PJ, Hazell JW. Treatment of tinnitus based on a neurophysiological model. In: Vernon JA, editor. Tinnitus treatment and relief. Needham Heights (MA): Allyn \& Bacon; 1998. p. 201-17.

32. Henry JA, Dennis KC, Schechter MA. General review of tinnitus: prevalence, mechanisms, effects, and management. J Speech Lang Hear Res. 2005;48(5):1204-35. [PMID:16411806] http://dx.doi.org/10.1044/1092-4388(2005/084)

33. Dobie RA. A review of randomized clinical trials in tinnitus. Laryngoscope. 1999;109(8):1202-11. [PMID:10443820] http://dx.doi.org/10.1097/00005537-199908000-00004

34. Meikle MB, Stewart BJ, Griest SE, Henry JA. Tinnitus outcomes assessment. Trends Amplif. 2008;12(3):223-35. [PMID:18599500] http://dx.doi.org/10.1177/1084713808319943

35. Schechter MA, Henry JA. Assessment and treatment of tinnitus patients using a "masking approach." J Am Acad Audiol. 2002;13(10):545-58. [PMID:12503923]

36. Vernon JA, Meikle MB. Tinnitus masking. In Tyler RS, editor. Tinnitus handbook. San Diego (CA): Singular Publishing Group; 2000. p. 313-56.

37. Jastreboff PJ. Phantom auditory perception (tinnitus): Mechanisms of generation and perception. Neurosci Res. 1990;8(4):221-54. [PMID:2175858] http://dx.doi.org/10.1016/0168-0102(90)90031-9

38. Jastreboff PJ, Hazell JW. Tinnitus retraining therapy: Implementing the neurophysiological model. New York (NY): Cambridge University Press; 2004.

39. Davis PB. Music and the acoustic desensitization protocol for tinnitus. In: Tyler RS, editor. Tinnitus treatment: Clinical protocols. New York (NY): Thieme Medical Publishers, Inc; 2006. p. 146-60.

40. Davis PB, Paki B, Hanley PJ. Neuromonics tinnitus treatment: Third clinical trial. Ear Hear. 2007;28(2):242-59.

\section{[PMID:17496674]}

http://dx.doi.org/10.1097/AUD.0b013e3180312619

41. Cima RF, Maes IH, Joore MA, Scheyen DJ, El Refaie A, Baguley DM, Anteunis LJ, van Breukelen GJ, Vlaeyen JW. Specialised treatment based on cognitive behaviour therapy versus usual care for tinnitus: A randomised controlled trial. Lancet. 2012;379(9830):1951-59. [PMID:22633033] http://dx.doi.org/10.1016/S0140-6736(12)60469-3

42. Henry JL, Wilson PH. The psychological management of chronic tinnitus. Needham Heights (MA): Allyn \& Bacon; 2001.

43. Searchfield GD. Hearing aids and tinnitus. In: Tyler RS, editor. Tinnitus treatment: Clinical protocols. New York (NY): Thieme; 2005. p. 161-75.

44. Shekhawat GS, Searchfield GD, Stinear CM. Role of hearing aids in tinnitus intervention: A scoping review. J Am Acad Audiol. 2013;24(8):747-62. [PMID:24131610] http://dx.doi.org/10.3766/jaaa.24.8.11

45. Hoare DJ, Adjamian P, Sereda M, Hall DA. Recent technological advances in sound-based approaches to tinnitus treatment: A review of efficacy considered against putative physiological mechanisms. Noise Health. 2013;15(63): 107-16. [PMID:23571301] http://dx.doi.org/10.4103/1463-1741.110292

46. Hoare DJ, Searchfield GD, Refaie AE, Henry JA. Sound therapy for tinnitus management: Practicable options. J Am Acad Audiol. 2014;25(1):62-75. [PMID:24622861] http://dx.doi.org/10.3766/jaaa.25.1.5

47. Henry JA, Zaugg TL, Schechter MA. Clinical guide for audiologic tinnitus management II: Treatment. Am J Audiol. 2005;14(1):49-70. [PMID:16180969] http://dx.doi.org/10.1044/1059-0889(2005/005)

48. Martinez-Devesa P, Perera R, Theodoulou M, Waddell A. Cognitive behavioural therapy for tinnitus. Cochrane Database Syst Rev. 2010;(9):CD005233. [PMID:20824844]

49. Hobson J, Chisholm E, El Refaie A. Sound therapy (masking) in the management of tinnitus in adults. Cochrane Database Syst Rev. 2010;(12):CD006371. [PMID:21154366]

50. Pichora-Fuller MK, Santaguida P, Hammill A, Oremus M, Westerberg B, Ali U, Patterson C, Raina P. Evaluation and treatment of tinnitus: Comparative effectiveness. Rockville (MD): Agency for Healthcare Research and Quality; 2013.

51. Henry JA, Zaugg TL, Myers PJ, Kendall CJ, Michaelides EM. A triage guide for tinnitus. J Fam Pract. 2010;59(7): 389-93. [PMID:20625568]

52. Myers PJ, Lorenz J, Weinel D. Written materials resource manual for patient health education. Tampa (FL): James A. Haley VA Patient Education Committee; 2003.

53. Henry JA, Zaugg TL, Myers PJ, Schechter MA. Using therapeutic sound with progressive audiologic tinnitus management. Trends Amplif. 2008;12(3):188-209. 
[PMID:18664499]

http://dx.doi.org/10.1177/1084713808321184

Submitted for publication August 28, 2013. Accepted in revised form December 23, 2013.

This article and any supplementary material should be cited as follows:

Myers PJ, Griest S, Kaelin C, Legro MW, Schmidt CJ,
Zaugg TL, Henry JA. Development of a progressive audiologic tinnitus management program for Veterans with tinnitus. J Rehabil Res Dev. 2014;51(4):609-22.

http://dx.doi.org/10.1682/JRRD.2013.08.0189

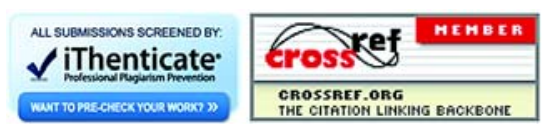


